\title{
A Note on the Newman-Unti Group and the BMS Charge Algebra in Terms of Newman-Penrose Coefficients
}

\author{
Glenn Barnich ${ }^{1,2}$ and Pierre-Henry Lambert ${ }^{1,2}$ \\ ${ }^{1}$ Physique Théorique et Mathématique, Université Libre de Bruxelles, \\ Campus Plaine, CP 231, 1050 Bruxelles, Belgium \\ 2 International Solvay Institutes, Campus Plaine, CP 231, 1050 Bruxelles, Belgium \\ Correspondence should be addressed to Pierre-Henry Lambert, pilamber@ulb.ac.be
}

Received 27 September 2012; Accepted 30 November 2012

Academic Editor: Andrei D. Mironov

Copyright (C) 2012 G. Barnich and P.-H. Lambert. This is an open access article distributed under the Creative Commons Attribution License, which permits unrestricted use, distribution, and reproduction in any medium, provided the original work is properly cited.

The symmetry algebra of asymptotically flat spacetimes at null infinity in four dimensions in the sense of Newman and Unti is revisited. As in the Bondi-Metzner-Sachs gauge, it is shown to be isomorphic to the direct sum of the abelian algebra of infinitesimal conformal rescalings with $\mathfrak{b m s}_{4}$. The latter algebra is the semidirect sum of infinitesimal supertranslations with the conformal Killing vectors of the Riemann sphere. Infinitesimal local conformal transformations can then consistently be included. We work out the local conformal properties of the relevant Newman-Penrose coefficients, construct the surface charges, and derive their algebra.

\section{Introduction}

The definitions of asymptotically flat four dimensional spacetimes at null infinity by Bondi et al. [1, 2] (BMS) and Newman-Unti (NU) [3] in 1962 merely differ by the choice of the radial coordinate. Such a change of gauge should not affect the asymptotic symmetry algebra if, as we contend, this concept is to have a major physical significance.

The problem of comparing the symmetry algebra in both cases is that, besides the difference in gauge, the very definitions of these algebras are not the same. Indeed, NU allow the leading part of the metric induced on Scri to undergo a conformal rescaling. When this generalization is considered in the BMS setting, it turns out that the symmetry algebra is the direct sum of the BMS algebra $\mathfrak{b m s}_{4}$ [4] with the abelian algebra of infinitesimal conformal rescalings [5,6]. There are two novel and independent aspects in this computation. 
(i) The first concerns the fact that the BMS algebra in 4 dimension involves the conformal Killing vectors of the unit, or equivalently, the Riemann sphere and can consistently accommodate infinitesimal local conformal transformations. The symmetry algebra $\mathfrak{b m s}_{4}$ then involves two commuting copies of the noncentrally extended Virasoro algebra, called superrotations in [7], and simultaneously the supertranslations generators are expanded in Laurent series. The standard, globally well-defined symmetry algebra $\mathfrak{b m s}_{4}^{\text {glob }}$ consists in restricting to the globally well-defined conformal Killing vectors of the sphere which correspond to infinitesimal Lorentz transformation, while the supertranslation generators are expanded into spherical harmonics.

This local versus global versions of the symmetry algebra are of course not related to the BMS gauge choice, but will also occur in alternative characterizations of the asymptotic symmetry algebra where the conformal Killing vectors of the sphere play a role. Examples of this are the geometrical approach of Geroch [8] based on Penrose's definition of null infinity [9] and also, as we will explicitly discuss in this paper, the asymptotic symmetries in the NU framework.

(ii) The second aspect is related to the modified Lie bracket that should be used when the vector fields parametrizing infinitesimal diffeomorphisms depend explicitly on the metric. Indeed, when using the modified Lie bracket, the spacetime vectors realize the asymptotic symmetry algebra everywhere in the bulk and furthermore, even on Scri, this bracket is needed to disentangle the algebra when conformal rescalings of the induced metric on Scri are allowed. Similarly, in the context of the AdS/CFT correspondence, this bracket allows one to realize the asymptotic symmetry algebra in the bulk and to disentangle the symmetry algebra at infinity when considering transformations that leave the FeffermanGraham ansatz invariant only up to conformal rescaling of the boundary metric [10]. From a mathematical point of view, the modified Lie bracket is the natural bracket of the Lie algebroid that is associated to any theory with gauge invariance [11].

What we will do in this paper is to rederive from scratch the asymptotic symmetry algebra in the NU framework by focusing on metric aspects and on the two novel features discussed above. As expected, the symmetry algebra is again the direct sum of $\mathfrak{b m s}_{4}$ with the abelian algebra of infinitesimal conformal rescalings of the metric on Scri and thus coincides, as it should, with the generalized symmetry algebra in the BMS approach. A related analysis of asymptotic symmetries in the NU context from the point of view of Scri and emphasizing global issues instead can be found in $[12,13]$.

Even though the results presented here are not really surprising in view of those in the BMS framework and the close relation between the NU and BMS approaches, the exercise of working out the details is justified because the NU framework is embedded in the context of the widely used Newman-Penrose formalism [14] so that explicit formulae in this context are directly relevant in many applications, see for example, the review article [15].

As a first application, we study the transformation properties of the NewmanPenrose coefficients parametrizing solution space in the NU approach. Our main focus is on the inhomogeneous terms in the transformation laws that contain the information on the central extensions of the theory. We then discuss the associated surface charges by following the analysis in the BMS gauge [16] and briefly compare with standard expressions that can be found in the literature. The algebra of these charges is derived and shown to involve field dependent central charges in the case of $\mathfrak{b m s}_{4}$ which vanish for $\mathfrak{b} \mathfrak{m s}_{4}^{\text {glob }}$. 


\section{NU Metric Ansatz for Asymptotically Flat Spacetimes}

The metric ansatz of NU is based on a family of null hypersurfaces labelled by the first coordinate, $x^{0} \equiv u=$ const. The second coordinate $x^{1} \equiv r$ is chosen as an affine parameter for the null geodesic generators $l^{\mu}$ of these hypersurfaces, so that $l^{\mu}=-\delta_{r}^{\mu}$. Up to a change of signature from $(+,-,-,-)$ to $(-,+,+,+)$, a renumbering of the indices, and the tetrad transformation that makes the conformal factor real, the line element considered in section 4 of NU [3] can be written as

$$
d s^{2}=W d u^{2}-2 d r d u+g_{A B}\left(d x^{A}-V^{A} d u\right)\left(d x^{B}-V^{B} d u\right)
$$

with associated inverse metric

$$
g^{\mu v}=\left(\begin{array}{ccc}
0 & -1 & 0 \\
-1 & -W & -V^{B} \\
0 & -V^{A} & g^{A B}
\end{array}\right)
$$

where

$$
g_{A B} d x^{A} d x^{B}=r^{2} \bar{\gamma}_{A B} d x^{A} d x^{B}+r C_{A B} d x^{A} d x^{B}+o(r)
$$

with $\bar{\gamma}_{A B}$ conformally flat. Below, we will use standard stereographic coordinates $\zeta=$ $\cot (\theta / 2) e^{i \phi}, \bar{\zeta}, \bar{\gamma}_{A B} d x^{A} d x^{B}=e^{2 \tilde{\varphi}} d \zeta d \bar{\zeta}, \tilde{\varphi}=\tilde{\varphi}(u, x)$. In particular, we use the notation $e^{2 \tilde{\varphi}}$ for the conformal factor. In Section 4 , we will give the explicit dictionary that allows one to translate to the quantities originally used by NU.

In addition, the choice of origin for the affine parameter of the null geodesics is fixed through the requirement that the term proportional to $r^{-2}$ in the expansion of the spin coefficient $-\rho=D_{\rho} l_{v} m^{\rho} \bar{m}^{v}$ is absent.

When expressed in terms of the metric, one finds

$$
\rho=-\frac{1}{4} g^{A B} g_{A B, r}=-\frac{1}{4} \partial_{r} \ln |g|=-r^{-1}+\frac{1}{4} C_{A}^{A} r^{-2}+o\left(r^{-2}\right),
$$

where $g=\operatorname{det} g_{\rho v}$ and the index has been raised with the inverse of $\bar{\gamma}_{A B}$. The requirement is thus equivalent to the condition

$$
C_{A}^{A}=0 .
$$

In the following we denote by $\bar{D}_{A}$ the covariant derivative with respect to $\bar{\gamma}_{A B}$ and by $\bar{\Delta}$ the associated Laplacian and by $\bar{R}$ the scalar curvature. In complex coordinates $\zeta, \bar{\zeta}, C_{\overline{\zeta \zeta}}=0$ and we define for later convenience $C_{\zeta \zeta}=e^{2 \tilde{\varphi}_{C}}, C_{\bar{\zeta} \bar{\zeta}}=e^{2 \tilde{\varphi}} \bar{C}$. Finally,

$$
V^{A}=O\left(r^{-2}\right), \quad W=-2 r \partial_{u} \tilde{\varphi}+\bar{\Delta} \tilde{\varphi}+O\left(r^{-1}\right),
$$

where $\bar{\Delta} \tilde{\varphi}=4 e^{-2 \tilde{\varphi}} \partial \bar{\partial} \tilde{\varphi}$ with $\partial=\partial_{\zeta}, \bar{\partial}=\partial_{\bar{\zeta}}$. 
The more restrictive fall-off conditions in [3] are relevant for integrating the field equations but play no role in the discussion of the asymptotic symmetry algebra.

\section{Asymptotic Symmetries in the NU Approach}

The infinitesimal NU transformations can be defined as those infinitesimal transformations that leave the form (2.2) and the fall-off conditions (2.3)-(2.6) invariant, up to a rescaling of the conformal factor $\delta \tilde{\varphi}\left(u, x^{A}\right)=\tilde{\omega}\left(u, x^{A}\right)$. In other words, they satisfy

$$
\begin{gathered}
\mathcal{L}_{\xi} g^{u u}=0, \quad \mathcal{L}_{\xi} g^{u A}=0, \quad \mathcal{L}_{\xi} g^{u r}=0, \\
\partial_{r}\left[\frac{1}{\sqrt{|g|}} \partial_{\rho}\left(\sqrt{|g|} \xi^{\rho}\right)\right]=o\left(r^{-2}\right), \\
\mathcal{L}_{\xi} g^{r A}=O\left(r^{-2}\right), \quad \mathcal{L}_{\xi} g^{A B}=-2 \tilde{\omega} g^{A B}+O\left(r^{-3}\right), \\
\mathcal{L}_{\xi} g^{r r}=2 r \partial_{u} \tilde{\omega}+2 \tilde{\omega} \bar{\Delta} \tilde{\varphi}-\bar{\Delta} \tilde{\omega}+O\left(r^{-1}\right) .
\end{gathered}
$$

Equations (3.1) are equivalent to

$$
\partial_{r} \xi^{v}=g^{\nu \rho} \partial_{\rho} \xi^{u} \Longleftrightarrow\left\{\begin{array}{l}
\partial_{r} \xi^{u}=0, \\
\partial_{r} \xi^{A}=\partial_{B} \xi^{u} g^{B A}, \\
\partial_{r} \xi^{r}=-\partial_{u} \xi^{u}-\partial_{A} \xi^{u} V^{A}
\end{array}\right.
$$

and are explicitly solved by

$$
\begin{gathered}
\xi^{u}=f, \\
\xi^{A}=Y^{A}+I^{A}, \quad I^{A}=-\partial_{B} f \int_{r}^{\infty} d r^{\prime} g^{A B}, \\
\xi^{r}=-r \partial_{u} f+Z+J, \quad J=\partial_{A} f \int_{r}^{\infty} d r^{\prime} V^{A},
\end{gathered}
$$

with $\partial_{r} f=0=\partial_{r} Y^{A}=\partial_{r} Z$. Equation (3.2) then implies

$$
Z=\frac{1}{2} \bar{\Delta} f
$$

The first equation of (3.3) requires $\partial_{u} Y^{A}=0$, the second that $Y^{A}$ is a conformal Killing vector of $\bar{\gamma}_{A B}$, which amounts to

$$
Y^{\zeta} \equiv Y=Y(\zeta), \quad Y^{\bar{\zeta}} \equiv \bar{Y}=\bar{Y}(\bar{\zeta})
$$


in the coordinates $\zeta, \bar{\zeta}$, and also that

$$
\partial_{u} f=f \partial_{u} \tilde{\varphi}+\frac{1}{2} \tilde{\psi},
$$

with $\psi=\bar{D}_{A} Y^{A}$, or more explicitly in $\zeta, \bar{\zeta}$ coordinates, $\psi=\partial Y+\bar{\partial} \bar{Y}+2 \Upsilon \partial \tilde{\varphi}+2 \bar{Y} \bar{\partial} \tilde{\varphi}$, and $\tilde{\psi}=\psi-2 \tilde{\omega}$. Finally, the last equation of (3.3) implies

$$
2\left(\partial_{u} Z+Z \partial_{u} \tilde{\varphi}\right)=Y^{A} \partial_{A} \bar{\Delta} \tilde{\varphi}+\psi \bar{\Delta} \tilde{\varphi}+2 \partial_{A} f \bar{\gamma}^{A B} \partial_{B} \partial_{u} \tilde{\varphi}+f \bar{\Delta} \partial_{u} \tilde{\varphi}-\bar{\Delta} \tilde{\omega},
$$

which is identically satisfied when taking the previous relations into account.

One approach is to consider that (3.8) fixes $\tilde{\omega}$ in terms of $f$ and $Y, \tilde{\omega}=(1 / 2) \psi+f \partial_{u} \tilde{\varphi}-$ $\partial_{u} f$. Consider Scri, the space $\supset$ with coordinates $u, \zeta, \bar{\zeta}$ and metric

$$
d s_{\partial}^{2}=0 d u^{2}+e^{2 \tilde{\varphi}} d \zeta d \bar{\zeta}
$$

The NU algebra is then defined as the commutator algebra of the vector fields

$$
\bar{\xi}=f \frac{\partial}{\partial u}+Y^{A} \frac{\partial}{\partial x^{A}},
$$

with $f=f\left(u, x^{A}\right)$ arbitrary and $Y^{A}(x)$ conformal Killing vectors of a conformally flat metric in 2 dimensions, or equivalently, the algebra of conformal vector fields of the degenerate metric (3.10).

This is not the symmetry algebra of asymptotically flat spacetimes in the sense of NU however. Indeed, $\tilde{\varphi}$ is arbitrary, it can for instance be considered as the finite ambiguity related to Penrose's conformal approach $[9,17,18]$ to null infinity. One can then interpret $\widetilde{\varphi}$ as part of the background structure, or in other words, of the gauge fixing [8], and compute the asymptotic symmetries for a fixed choice of $\tilde{\varphi}$, that is, $\widetilde{\omega}=0$ in the formulae above, or ask the more general question of how the asymptotic symmetries depend on changes in $\tilde{\varphi}$ by an arbitrary infinitesimal amount $\tilde{\omega}$. In both cases, one has to consider (3.8) as a differential equation for $f$. As we now show, the symmetry algebra will then be isomorphic to the trivially extended $\mathfrak{b m s}_{4}$ algebra by the abelian algebra of infinitesimal conformal rescalings, as it should, and as a consequence, the Poincare algebra is embedded therein in a natural way. Furthermore, there is a natural realization of the asymptotic symmetry algebra on an asymptotically flat 4 dimensional bulk spacetime. Note also that, for $\tilde{\omega}=0,(3.8)$ has been interpreted from the point of view of Penrose's conformal approach to null infinity in [12] following [19] and related to the preservation of null angles, which is the standard way $[9,17,20,21]$ to recover the BMS algebra from geometrical data on Scri.

The general solution for (3.8) reads

$$
f=e^{\tilde{\varphi}}\left[\tilde{T}+\frac{1}{2} \int_{0}^{u} d u^{\prime} e^{-\tilde{\varphi}} \widetilde{\varphi}\right], \quad \tilde{T}=\tilde{T}(\zeta, \bar{\zeta}),
$$


and the general solution to (3.1)-(3.3) defining the asymptotic symmetries is given by $\xi^{\rho}$ as in (3.5) where $Z, Y^{A}, f$ satisfy (3.6), (3.7), (3.12) with $\widetilde{\omega}$ arbitrary. Asymptotic Killing vectors thus depend on $Y^{A}, \widetilde{T}, \tilde{\omega}$ and the metric, $\xi=\xi[Y, \widetilde{T}, \tilde{\omega} ; g]$.

For such metric-dependent vector fields, consider on the one hand the suitably modified Lie bracket taking the metric dependence of the spacetime vectors into account,

$$
\left[\xi_{1}, \xi_{2}\right]_{M}=\left[\xi_{1}, \xi_{2}\right]-\delta_{\xi_{1}}^{g} \xi_{2}+\delta_{\xi_{2}}^{g} \xi_{1}
$$

where $\delta_{\xi_{1}}^{g} \xi_{2}$ denotes the variation in $\xi_{2}$ under the variation of the metric induced by $\xi_{1}, \delta_{\xi_{1}}^{g} g_{\mu \nu}=$ $\mathcal{L}_{\xi_{1}} g_{\mu \nu}$.

Consider on the other hand the extended $\mathfrak{b m s}_{4}$ algebra, that is, the semidirect sum of the algebra of conformal Killing vectors of the Riemann sphere with the abelian ideal of infinitesimal supertranslations, trivially extended by infinitesimal conformal rescalings of the conformally flat degenerate metric on Scri. More explicitly, the commutation relations are given by $\left[\left(Y_{1}, \tilde{T}_{1}, \tilde{\omega}_{1}\right),\left(Y_{2}, \tilde{T}_{2}, \tilde{\omega}_{2}\right)\right]=(\widehat{Y}, \widehat{\widetilde{T}}, \widehat{\tilde{\omega}})$ where

$$
\begin{gathered}
\hat{\Upsilon}^{A}=Y_{1}^{B} \partial_{B} Y_{2}^{A}-Y_{2}^{B} \partial_{B} Y_{1}^{A}, \\
\widehat{\tilde{T}}=Y_{1}^{A} \partial_{A} \widetilde{T}_{2}-Y_{2}^{A} \partial_{A} \widetilde{T}_{1}+\frac{1}{2}\left(\widetilde{T}_{1} \partial_{A} Y_{2}^{A}-\widetilde{T}_{2} \partial_{A} Y_{1}^{A}\right), \\
\widehat{\tilde{\omega}}=0 .
\end{gathered}
$$

It thus follows the following.

Theorem 3.1. The spacetime vectors $\xi[Y, \widetilde{T}, \tilde{\omega} ; g]$ realize the extended $\mathfrak{b m s}_{4}$ algebra in the modified Lie bracket,

$$
\left[\xi\left[Y_{1}, \tilde{T}_{1}, \tilde{\omega}_{1} ; g\right], \xi\left[Y_{2}, \tilde{T}_{2}, \tilde{\omega}_{2} ; g\right]\right]_{M}=\xi[\widehat{Y}, \widehat{\widetilde{T}}, \widehat{\tilde{\omega}} ; g]
$$

in the bulk of an asymptotically flat spacetime in the sense of Newman and Unti.

Note in particular that for two different choices of the conformal factor $\tilde{\varphi}$ which is held fixed, $\tilde{\omega}=0$, the asymptotic symmetry algebras are isomorphic to $\mathfrak{b m s}_{4}$, which is thus a gauge invariant statement.

Proof. The proof follows closely the one in [6] for the BMS gauge. In order to be self-contained we recall the different steps here. In a first stage, one shows that on $\supset$, the vectors fields $\bar{\xi}[Y, \widetilde{T}, \tilde{\omega} ; \bar{\gamma}]$ given in (3.11) with $f$ as in (3.12) realize the extended $\mathfrak{b m s}_{4}$ algebra in terms of the modified Lie bracket. Indeed, this is obvious for the $A$ components which do not depend on the metric so that the modified bracket reduces to the standard Lie bracket for these components. For the $u$ component, taking into account that

$$
\delta_{\bar{\xi}_{1}}^{g} f_{2}=\tilde{\omega}_{1} f_{2}+\frac{1}{2} e^{\tilde{\varphi}} \int_{0}^{u} d u^{\prime} e^{-\widetilde{\varphi}}\left[-\tilde{\omega}_{1}\left(\psi_{2}-2 \tilde{\omega}_{2}\right)+2 Y_{2}^{A} \partial_{A} \tilde{\omega}_{1}\right]
$$


we have $\left.\left[\bar{\xi}_{1}, \bar{\xi}_{2}\right]_{M}^{u}\right|_{u=0}=\left.e^{\tilde{\varphi}}\right|_{u=0} \widehat{T}$. Direct computation then shows that $\partial_{u}\left(\left[\bar{\xi}_{1}, \bar{\xi}_{2}\right]_{M}^{u}\right)=\widehat{f} \partial_{u} \tilde{\varphi}+$ $(1 / 2) \bar{D}_{A} \widehat{Y}^{A}$ with $\hat{f}$ given by (3.12) with $\tilde{T}, Y, \tilde{\omega}$ replaced by their hatted counterparts, implying the result for the $u$ component.

For the spacetime vectors, direct computation gives $\left[\xi_{1}, \xi_{2}\right]_{M}^{u}=\left[\bar{\xi}_{1}, \bar{\xi}_{2}\right]_{M}^{u}=\widehat{f}$. Using the defining property (3.4), one then finds that $\partial_{r}\left(\left[\xi_{1}, \xi_{2}\right]_{M}^{\rho}\right)=g^{\rho v} \partial_{v} \hat{f}$. For the $A$ components the result then follows from the one on $\supset, \lim _{r \rightarrow \infty}\left[\xi_{1}, \xi_{2}\right]_{M}^{A}=\widehat{Y}^{A}$. This is due to the fact that $I^{A}$ goes to zero at infinity, that the nonvanishing term at infinity does not involve the metric, and that the correction term in the bracket does not change the asymptotic behaviour. Finally, for the $r$ component, we still need to check that the $r$ independent component of $\left[\xi_{1}, \xi_{2}\right]_{M}^{r}$ is given by $(1 / 2) \bar{\Delta} \hat{f}$, which follows by direct computation.

For completeness, let us also stress here that, if one focuses on local properties and expands the conformal Killing vectors $Y^{A} \partial_{A}$ and the infinitesimal supertranslations $T$ in Laurent series,

$$
\begin{gathered}
l_{n}=-\zeta^{n+1} \frac{\partial}{\partial \zeta^{\prime}} \quad \bar{l}_{n}=-\bar{\zeta}^{n+1} \frac{\partial}{\partial \bar{\zeta}^{\prime}} \quad n \in \mathbb{Z}, \\
\tilde{T}_{m, n}=\zeta^{m} \bar{\zeta}^{n}, \quad m, n \in \mathbb{Z},
\end{gathered}
$$

the commutation relations for the complexified $\mathfrak{b m s}_{4}$ algebra read

$$
\begin{gathered}
{\left[l_{m}, l_{n}\right]=(m-n) l_{m+n}, \quad\left[\bar{l}_{m}, \bar{l}_{n}\right]=(m-n) \bar{l}_{m+n}, \quad\left[l_{m}, \bar{l}_{n}\right]=0,} \\
{\left[l_{l}, T_{m, n}\right]=\left(\frac{l+1}{2}-m\right) T_{m+l, n}, \quad\left[\bar{l}_{l}, T_{m, n}\right]=\left(\frac{l+1}{2}-n\right) T_{m, n+l} .}
\end{gathered}
$$

The $\mathfrak{b m s}_{4}$ algebra contains as subalgebra the Poincare algebra, which we identify with the algebra of exact Killing vectors of the Minkowski metric equipped with the standard Lie bracket. It is spanned by the generators

$$
l_{-1}, l_{0}, l_{1}, \quad \bar{l}_{-1}, \bar{l}_{0}, \bar{l}_{1}, \quad \widetilde{T}_{0,0}, \widetilde{T}_{1,0}, \widetilde{T}_{0,1}, \widetilde{T}_{1,1}
$$

Nontrivial central extensions of the algebra (3.18) have been studied in [7]: the computation of $H^{2}\left(\mathfrak{b m s}_{4}\right)$ reveals that there is only the standard ones for the Virasoro algebra extending the first two commutation relations.

\section{Explicit Relation between the NU and the BMS Gauges}

The definition of asymptotically flat spacetimes in the BMS approach $[1,2,4]$ as reviewed in $[5,6]$ amounts to replacing $g_{u u}=1 / g^{u u}=-1$ by

$$
g_{u u}=1 / g^{u u}=-e^{2 \beta}, \quad \beta=O\left(r^{-2}\right)
$$


in (2.1) and (2.2) while imposing the additional requirement that

$$
\operatorname{det} g_{A B}=r^{4} \operatorname{det} \bar{\gamma}_{A B}
$$

Both definitions then differ just by a choice of radial coordinate. Indeed, replacing the radial coordinate by a function of the 4 coordinates preserves the zeros in (2.1) and (2.2) (see e.g., the discussion in [22]). Furthermore, for first nontrivial order in $r$, the determinant condition leads to the same restriction (2.5) as the choice of the origin of the affine parameter. It follows that the relation between the two radial coordinates does not involve constant terms and is of the form

$$
r^{\prime}=r+O\left(r^{-1}\right)
$$

More explicitly, starting from the NU approach, BMS coordinates are obtained by defining the new radial coordinates as [23]

$$
r_{\mathrm{BMS}}=\left(\frac{\operatorname{det} g_{A B}}{\operatorname{det} \bar{\gamma}_{A B}}\right)^{1 / 4}
$$

Conversely, starting from the BMS approach with radial coordinate $r$, NU coordinates are obtained by changing the radial coordinate to

$$
r_{N}=r-\int_{r}^{\infty} d r^{\prime}\left(e^{2 \beta}-1\right)
$$

These changes of coordinates only affect lower-order terms in the asymptotic expansion of the metric that plays no role in the definition of asymptotic symmetries and explains a posteriori why the asymptotic symmetry algebras in both approaches are isomorphic.

At this stage, the dynamics of the theory comes into play. The Einstein equations are solved order by order in $r$. In the first orders, there are integrations "constants" that appear as free data characterizing asymptotically flat solutions. We will now work out the explicit relation between these data in both approaches. The inverse metric in the BMS gauge (as discussed in [6]) is given by

$$
\begin{gathered}
g_{\mathrm{BMS}}^{\mu \nu}=\left(\begin{array}{ccc}
0 & -e^{-2 \beta} & 0 \\
-e^{-2 \beta} & -e^{-2 \beta} \frac{V}{r} & -e^{-2 \beta} U^{B} \\
0 & -e^{-2 \beta} U^{A} & g^{A B}
\end{array}\right), \\
g_{A B}=r^{2} \bar{\gamma}_{A B}+r C_{A B}+\frac{1}{4} \bar{\gamma}_{A B} C_{D}^{C} C_{C}^{D}+O\left(r^{-1}\right) .
\end{gathered}
$$


For simplicity, we assume here that there is no trace-free part $D_{A B}$ at order 0 and that the conformal factor is time-independent, $\partial_{u} \tilde{\varphi}=0$, in which case the news tensor is simply $N_{A B}=$ $\partial_{u} C_{A B}$ and $f=T+(1 / 2) u \widetilde{\varphi}$ with $T=e^{\tilde{\varphi}} \widetilde{T}$. Writing

$$
C_{\zeta \zeta}=e^{2 \tilde{\varphi}_{C}}, \quad C_{\bar{\zeta} \bar{\zeta}}=e^{2 \tilde{\varphi}} \bar{C}, \quad C_{\overline{\zeta \zeta}}=0,
$$

we have

$$
\begin{gathered}
\beta=-\frac{1}{4} r^{-2} c \bar{c}+O\left(r^{-4}\right), \\
U^{\zeta}=-\frac{2}{r^{2}} e^{-4 \tilde{\varphi}} \partial\left(e^{22 \tilde{\varphi}} \bar{c}\right)-\frac{2}{3 r^{3}}\left[N^{\zeta}-4 e^{-4 \tilde{\varphi}} \bar{c} \bar{\partial}\left(e^{2 \tilde{\varphi}} c\right)\right]+O\left(r^{-4}\right), \\
\frac{V}{r}=4 e^{-2 \tilde{\varphi}} \partial \bar{\partial} \tilde{\varphi}+r^{-1} 2 M+O\left(r^{-2}\right),
\end{gathered}
$$

which implies in particular that

$$
r_{N}=r+\frac{c \bar{c}}{2 r}+O\left(r^{-3}\right)
$$

The only consequence of Einstein's equations on the angular momentum and mass aspects $N^{\zeta}=N^{\zeta}(u, \zeta, \bar{\zeta}), M=M(u, \zeta, \bar{\zeta})$ is the evolution equations

$$
\begin{gathered}
\partial_{u} M=-\frac{1}{8} N_{B}^{A} N_{A}^{B}+\frac{1}{8} \bar{\Delta} \bar{R}+\frac{1}{4} \bar{D}_{A} \bar{D}_{C} N^{C A} \\
\partial_{u} N_{A}=\partial_{A} M+\frac{1}{4} C_{A}^{B} \partial_{B} \bar{R}+\frac{1}{16} \partial_{A}\left[N_{C}^{B} C_{B}^{C}\right]-\frac{1}{4} \bar{D}_{A} C_{B}^{C} N_{C}^{B} \\
-\frac{1}{4} \bar{D}_{B}\left[C_{C}^{B} N_{A}^{C}-N_{C}^{B} C_{A}^{C}\right]-\frac{1}{4} \bar{D}_{B}\left[\bar{D}^{B} \bar{D}_{C} C_{A}^{C}-\bar{D}_{A} \bar{D}_{C} C^{B C}\right] .
\end{gathered}
$$

Consider now the "eth" operators [24] defined here for a field $\eta^{s}$ of spin weight $s$ according to the conventions of [25] through

$$
\widetilde{\partial} \eta^{s}=P^{1-s} \bar{\partial}\left(P^{s} \eta^{s}\right), \quad \bar{\partial} \eta^{s}=P^{1+s} \partial\left(P^{-s} \eta^{s}\right), \quad P=\sqrt{2} e^{-\widetilde{\varphi}},
$$

where $\check{\partial}, \bar{\partial}$ raise, respectively, lower the spin weight by one unit and satisfy

$$
[\bar{ळ}, \precsim] \eta^{s}=\frac{s}{2} \bar{R} \eta^{s}
$$

The spin weights of the various quantities are summarized in Table 1 . Note that the $P$ used here differs from the one used in [3], which we will denote by $P_{N}$ below. It also no longer denotes the particular function $(1 / 2)(1+\zeta \bar{\zeta})$, contrary to the notation used in $[6,16]$.

In order to compare with the notation used in [3], we use $\zeta=x^{3}+i x^{4}$. With $x^{\prime \alpha}=u, r_{\mathrm{N}}, x^{3}, x^{4}$ and $x^{\mu}=u, r, \zeta, \bar{\zeta}$, computing $g_{\mathrm{N}}^{\alpha \beta}\left(x^{\prime}\right)=-\left(\left(\partial x^{\prime \alpha} / \partial x^{\mu}\right) g_{\mathrm{BMS}}^{\mu \nu}\left(\partial x^{\prime \beta} / \partial x^{\nu}\right)\right)\left(x\left(x^{\prime}\right)\right)$, 
Table 1: Spin and conformal weights.

\begin{tabular}{lcccccccc}
\hline & $\sigma^{0}$ & $\dot{\sigma}^{0}$ & $\Psi_{4}^{0}$ & $\Psi_{3}^{0}$ & $\Psi_{2}^{0}$ & $\Psi_{1}^{0}$ & $y$ & $T$ \\
\hline$S$ & 2 & 2 & -2 & -1 & 0 & 1 & -1 & 0 \\
$w$ & -1 & -2 & -3 & -3 & -3 & -3 & 1 & 1 \\
\hline
\end{tabular}

where the overall minus sign takes the change of signature into account, then gives the following dictionary by comparing with [3]:

$$
\begin{gathered}
P_{N}=\frac{1}{\sqrt{2}} e^{-\tilde{\varphi}}=\frac{1}{2} P, \quad \nabla=2 \bar{\partial}, \quad \mu^{0}=-P^{2} \partial \bar{\partial} \ln P=\frac{1}{2} \bar{\Delta} \tilde{\varphi}=-\frac{1}{4} \bar{R}, \\
\Psi_{2}^{0}+\bar{\Psi}_{2}^{0}=-2 M-\partial_{u}(c \bar{c}), \quad \sigma^{0}=\bar{c}, \quad \omega^{0}=\bar{\partial} \sigma^{0}, \\
\Psi_{1}^{0}=-P N_{\bar{\zeta}}-\sigma^{0} \partial \bar{\sigma}^{0}-\frac{3}{4} \partial\left(\sigma^{0} \bar{\sigma}^{0}\right) .
\end{gathered}
$$

For convenience, let us also use

$$
\Psi_{3}^{0}=-ð \dot{\bar{\sigma}}^{0}-\frac{1}{4} \bar{\partial} \bar{R}, \quad \Psi_{4}^{0}=-\ddot{\bar{\sigma}}^{0} .
$$

In these terms,

$$
\dot{\Psi}_{3}^{0}=ð \Psi_{4}^{0}, \quad \dot{\Psi}_{2}^{0}=ð \Psi_{3}^{0}+\sigma^{0} \Psi_{4}^{0}, \quad \dot{\Psi}_{1}^{0}=ð \Psi_{2}^{0}+2 \sigma^{0} \Psi_{3}^{0}
$$

Indeed, the first equation holds by definition and the assumed time-independence of $P$. The evolution equation (4.10) is equivalent to the real part of the second equation. Taking into account the on-shell relation of the NU framework,

$$
\Psi_{2}^{0}-\bar{\Psi}_{2}^{0}=\overline{\check{\sigma}}^{2} \sigma^{0}-\partial^{2} \bar{\sigma}^{0}+\bar{\sigma}^{0} \dot{\sigma}^{0}-\sigma^{0} \dot{\bar{\sigma}}^{0}
$$

we find

$$
M=-\Psi_{2}^{0}-\sigma^{0} \dot{\bar{\sigma}}^{0}+\frac{1}{2} \bar{\partial}^{2} \sigma^{0}-\frac{1}{2} ð^{2} \bar{\sigma}^{0}
$$

in terms of which (4.10) is fully equivalent to the second equation of (4.16) and (4.11) is equivalent to the last equation of (4.16), in agreement with [3]. 


\section{Transformation Laws of the NU Coefficients Characterizing Asymptotic Solutions}

Let $y=P^{-1} \bar{Y}$ and $\bar{y}=P^{-1} Y$. The conformal Killing equations and the conformal factor then become

$$
\partial \bar{y}=0=\bar{\partial} y, \quad \psi=(\partial y+\bar{\partial} \bar{y}) .
$$

It follows for instance that

$$
\bar{\partial} \partial y=-\frac{\bar{R}}{2} y, \quad \partial^{2} \psi=\partial^{3} y-\frac{1}{2} \bar{y} \partial \bar{R}, \quad \bar{\partial} \partial \psi=-\frac{1}{2}[ð(\bar{R} y)+\bar{\partial}(\bar{R} \bar{y})] .
$$

Using the notation $S=(Y, \widetilde{T}, \tilde{\omega})$, we have $-\delta_{S} \bar{\gamma}_{A B}=2 \tilde{\omega} \bar{\gamma}_{A B}$ for the background metric and

$$
\left[-\delta_{S}, \bar{\partial}\right] \eta^{s}=-\tilde{\omega} \bar{\partial} \eta^{s}+s \bar{\delta} \tilde{\omega} \eta^{s}, \quad\left[-\delta_{S}, \widetilde{\partial}\right] \eta^{s}=-\tilde{\omega} \partial \eta^{s}-s \widetilde{\partial} \tilde{\omega} \eta^{s} .
$$

To work out the transformation properties of the NU coefficients characterizing asymptotic solution space, one needs to evaluate the subleading terms in $\mathcal{L}_{\xi} g_{N}^{\alpha \beta}$ on-shell. This can also be done by translating the results from the BMS gauge, which yields

$$
\begin{aligned}
& -\delta_{S} \sigma^{0}=\left[f \partial_{u}+y \partial+\bar{y} \bar{\partial}+\frac{3}{2} \partial y-\frac{1}{2} \bar{\partial} \bar{y}-\tilde{w}\right] \sigma^{0}-\partial^{2} f, \\
& -\delta_{S} \dot{\sigma}^{0}=\left[f \partial_{u}+y \partial+\bar{y} \bar{\partial}+2 \partial y-2 \tilde{\omega}\right] \dot{\sigma}^{0}-\frac{1}{2} \partial^{2} \widetilde{\psi}, \\
& -\delta_{S} \Psi_{4}^{0}=\left[f \partial_{u}+y \partial+\bar{y} \bar{\partial}+\frac{1}{2} \partial y+\frac{5}{2} \bar{\partial} \bar{y}-3 \tilde{w}\right] \Psi_{4}^{0}, \\
& -\delta_{S} \Psi_{3}^{0}=\left[f \partial_{u}+y \partial+\bar{y} \bar{\partial}+\partial y+2 \bar{\partial} \bar{y}-3 \tilde{\omega}\right] \Psi_{3}^{0}+\partial f \Psi_{4}^{0} \\
& -\delta_{S} \Psi_{2}^{0}=\left[f \partial_{u}+y \partial+\bar{y} \bar{\partial}+\frac{3}{2} \partial y+\frac{3}{2} \bar{\partial} \bar{y}-3 \tilde{w}\right] \Psi_{2}^{0}+2 \partial f \Psi_{3}^{0} \\
& -\delta_{S} \Psi_{1}^{0}=\left[f \partial_{u}+y \partial+\bar{y} \bar{\partial}+2 \partial y+\bar{\partial} \bar{y}-3 \tilde{\omega}\right] \Psi_{1}^{0}+3 \partial f \Psi_{2}^{0} .
\end{aligned}
$$

Following for instance the terminology in [26] Section 3, but now for general infinitesimal transformations $\zeta^{\prime}=\zeta+\epsilon Y(\zeta), \bar{\zeta}^{\prime}=\bar{\zeta}+\epsilon \bar{Y}(\bar{\zeta})$ instead of those associated to linear fractional transformations on the sphere and also considering $\bar{\zeta}$ as the holomorphic coordinate instead of $\zeta$, a field $\eta$ has spin weight $s$ and conformal weight $w$ if it transforms as

$$
-\delta_{Y, \bar{Y}} \eta=\left[Y^{A} \partial_{A}+\frac{s}{2}(\bar{\partial} \bar{Y}-\partial Y)-\frac{w}{2} \psi\right] \eta
$$


Table 2: Rank and density weights.

\begin{tabular}{ccccccccc}
\hline & $P^{-1} \sigma^{0}$ & $P^{-2} \dot{\sigma}^{0}$ & $P^{-3} \Psi_{4}^{0}$ & $P^{-3} \Psi_{3}^{0}$ & $P^{-3} \Psi_{2}^{0}$ & $P^{-3} \Psi_{1}^{0}$ & $\bar{Y}$ & $\tilde{T}$ \\
\hline$S$ & 2 & 2 & -2 & -1 & 0 & 1 & -1 & 0 \\
$n$ & $-1 / 2$ & 0 & $1 / 2$ & 1 & $3 / 2$ & 1 & -1 & $-1 / 2$ \\
\hline
\end{tabular}

A tensor density of rank $s \geqslant 0$ and weight $n$ transforms as

$$
-\delta_{Y, \bar{Y}} A_{\bar{\zeta} \ldots \bar{\zeta}}=\left[Y^{A} \partial_{A}+s \bar{\partial} \bar{Y}+n(\partial Y+\bar{\partial} \bar{Y})\right] A_{\bar{\zeta} \cdots \overline{\zeta^{\prime}}}
$$

while for rank $s \leqslant 0$ and weight $n$, we have

$$
-\delta_{Y, \bar{Y}} A_{\zeta \cdots \zeta}=\left[Y^{A} \partial_{A}-s \partial Y+n(\partial Y+\bar{\partial} \bar{Y})\right] A_{\zeta \cdots \zeta}
$$

It then follows that a tensor density of weights $(s, n)$ defines a field of weights $(s,-(2 n+|s|))$ and conversely, a field of weights $(s, w)$ defines a tensor density of weights $(s,-(1 / 2)(w+|s|))$. For $s \geqslant 0$, this is done through $\eta=A_{\bar{\zeta} \cdots \bar{\zeta}} P^{2 n+s}$ and $A_{\bar{\zeta} \cdots \bar{\zeta}}=P^{w} \eta$. For $s \leqslant 0$, we have $\eta=$ $A_{\zeta \cdots \zeta} P^{2 n-s}$ and $A_{\zeta \cdots \zeta}=P^{w} \eta$. Note that complex conjugation gives rise to opposite spin weight and rank but leaves the conformal and density weights unchanged. Alternatively, (5.5) can be written as

$$
-\delta_{y, \bar{y}} \eta=\left[y \partial+\bar{y} \bar{\partial}+\frac{s-w}{2} ð y-\frac{s+w}{2} \bar{\partial} \bar{y}\right] \eta
$$

When focusing on $T=0=\widetilde{\omega}$ at the surface $u=0$ and on the homogeneous part of the transformations, this gives the weights summarized in Tables 1 and 2 . These tables are extended to the Lie algebra elements, which are passive in all our computations, by writing $[Y, \tilde{T}]=-\delta_{Y, \bar{Y}} \tilde{T}$ and $\left[Y, Y^{\prime}\right]^{A}=-\delta_{Y, \bar{Y}} Y^{\prime} A$.

\section{Surface Charge Algebra}

In this section, $\tilde{w}=0$ so that $f=T+(1 / 2) u \psi$ and we use the notation $s=(y, \bar{y}, T)$ for elements of the symmetry algebra, which is given in these terms by $\left[s_{1}, s_{2}\right]=\widehat{s}$ where

$$
\begin{gathered}
\widehat{y}=y_{1} \partial y_{2}-(1 \longleftrightarrow 2), \quad \hat{\bar{y}}=\bar{y}_{1} \bar{\partial} \bar{y}_{2}-(1 \longleftrightarrow 2), \\
\widehat{T}=\left(y_{1} \partial+\bar{y}_{1} \bar{\partial}\right) T_{2}-\frac{1}{2} \psi_{1} T_{2}-(1 \longleftrightarrow 2) .
\end{gathered}
$$


The translation of the charges, the nonintegrable piece due to the news, and the central charges computed in [16] is given here

$$
\begin{gathered}
Q_{s}[X]=-\frac{1}{8 \pi G} \int d^{2} \Omega^{\varphi}\left[\left(f\left(\Psi_{2}^{0}+\sigma^{0} \dot{\bar{\sigma}}^{0}\right)+y\left(\Psi_{1}^{0}+\sigma^{0} \partial \bar{\sigma}^{0}+\frac{1}{2} \partial\left(\sigma^{0} \bar{\sigma}^{0}\right)\right)\right)+\text { c.c. }\right], \\
\Theta_{s}[\delta x, x]=\frac{1}{8 \pi G} \int d^{2} \Omega^{\varphi} f\left[\dot{\bar{\sigma}}^{0} \delta \sigma^{0}+\text { c.c. }\right] \\
K_{s_{1}, s_{2}}[X]=\frac{1}{8 \pi G} \int d^{2} \Omega^{\varphi}\left[\left(\frac{1}{4} f_{1} \partial f_{2} \overline{\partial R}+\frac{1}{2} \bar{\sigma}^{0} f_{1} \partial^{2} \psi_{2}-(1 \longleftrightarrow 2)\right)+\text { c.c. }\right] .
\end{gathered}
$$

Note that one could also write the charges $Q_{s}[x]$ by allowing for the additional terms $\left((1 / 2) \Im^{2} \bar{\sigma}^{0}-(1 / 2) \bar{\partial}^{2} \sigma^{0}\right)$ in the first parenthesis since these terms were cancelled with the corresponding terms in the complex conjugate expression. Note also that not $\Psi_{2}^{0}$ but only $\Psi_{2}^{0}+\bar{\Psi}_{2}^{0}$ is free data on-shell because of the relation (4.17).

We recognize all the ingredients of the surface charges described in [27], which in turn have been related there to previous expressions in the literature and, in particular, to the twistorial approach of Penrose [28]. More precisely, up to conventions, $Q_{0,0, T}$ agrees with Geroch's linear supermomentum [8] $Q_{g n}+\bar{Q}_{g n}$, as given in (A1.12) of [27]. The angular (super-)momentum that we get is

$$
Q_{y, 0,0}=-\frac{1}{8 \pi G} \int d^{2} \Omega^{\varphi} y\left[\Psi_{1}^{0}+\sigma^{0} \partial \bar{\sigma}^{0}+\frac{1}{2} ð\left(\sigma^{0} \bar{\sigma}^{0}\right)-\frac{u}{2} \partial\left(\Psi_{2}^{0}+\bar{\Psi}_{2}^{0}+\partial_{u}\left(\sigma^{0} \bar{\sigma}^{0}\right)\right)\right] .
$$

It differs from $Q_{\eta_{c}}$ given in (4) of [27] by the explicitly $u$-dependent term of the second line. It thus has a similar structure to Penrose's angular momentum as described in (11), (12), and (17a) of [27] in the sense that it also differs by a specific amount of linear supermomentum, but the amount is different and explicitly $u$-dependent,

$$
Q_{y, 0,0}=Q_{y, 0,0}^{u=0}+\frac{1}{2} u Q_{0,0, \partial y} .
$$

The main result derived in [16] states that

(i) if one is allowed to integrate by parts,

$$
\int d^{2} \Omega^{\varphi} \widetilde{\partial} \eta^{-1}=0=\int d^{2} \Omega^{\varphi} \bar{\partial} \eta^{1},
$$

where $d^{2} \Omega^{\varphi}=(2 d \zeta \wedge d \bar{\zeta}) / i P^{2}$,

(ii) if one defines the "Dirac bracket" through

$$
\left\{Q_{s_{1}}, Q_{s_{2}}\right\}^{*}[x]=-\delta_{s_{2}} Q_{s_{1}}[x]+\Theta_{s_{2}}\left[-\delta_{s_{1}} x, x\right],
$$


then the charges define a representation of the $\mathfrak{b m s}_{4}$ algebra, up to a field-dependent central extension,

$$
\left\{Q_{s_{1}}, Q_{s_{2}}\right\}^{*}=Q_{\left[s_{1}, s_{2}\right]}+K_{s_{1}, s_{2}}
$$

where $K_{s_{1}, s_{2}}$ satisfies the generalized cocycle condition

$$
K_{\left[s_{1}, s_{2}\right], s_{3}}-\delta_{s_{3}} K_{s_{1}, s_{2}}+\operatorname{cyclic}(1,2,3)=0 .
$$

The representation theorem contained in (6.7) and (6.8) can be verified directly in the present context by starting from (6.2), (4.17) and using the properties (4.13), (6.5) of $\partial$, the evolution equations (4.16), the conformal Killing equations (5.1), the $\mathfrak{b m s}_{4}$ algebra (6.1), and the transformation laws (5.4).

Several remarks are in order as follows.

(i) Integrations by parts are justified for regular functions on the sphere and thus for $\mathfrak{b m s}_{4}^{\text {glob }}$ and regular solutions. In the case of Laurent series more care is needed, see for example, [29]. We will address this question elsewhere.

(ii) For the globally well-defined $\mathfrak{b m s}_{4}^{\text {glob }}$ algebra on the sphere, the central charge $K_{s_{1}, s_{2}}$ vanishes.

(iii) The nonconservation of the charges follows by taking $s_{2}=(0,0,1)$ and $s_{1}=s$. Indeed, since $(d / d u) Q_{s}=(\partial / \partial u) Q_{s}-\delta_{(0,0,1)} Q_{s}$, the equality of the right hand sides of (6.6) and (6.7) gives

$$
\frac{d}{d u} Q_{s}=-\frac{1}{8 \pi G} \int d^{2} \Omega^{\varphi}\left[\dot{\bar{\sigma}}^{0}\left(-\delta_{s} \sigma^{0}\right)+\frac{1}{4} \partial f \bar{\partial} \bar{R}+\frac{1}{2} \bar{\sigma}^{0} \check{\partial}^{2} \psi+\text { c.c. }\right] .
$$

For $s=(0,0,1)$, this gives the standard Bondi-Sachs mass loss formula,

$$
\frac{d}{d u} Q_{0,0,1}=-\frac{1}{8 \pi G} \int d^{2} \Omega^{\varphi}\left[\dot{\bar{\sigma}}^{0} \dot{\sigma}^{0}+\text { c.c. }\right] \text {. }
$$

It also follows that the standard $\mathfrak{b m s}_{4}^{\text {glob }}$ charges are all conserved on the sphere in the absence of news.

To the best of our knowledge, except for the previous analysis in the BMS gauge, the above representation result does not exist elsewhere in the literature. A more detailed discussion of its implications, a detailed comparison with results in the literature as well as a self-contained derivation of the $\mathfrak{b m s}_{4}$ transformation laws in the context of the NewmanPenrose formalism will be given elsewhere.

\section{Acknowledgments}

The authors thank Cédric Troessaert for useful discussions. This work is supported in part by the Fund for Scientific Research-FNRS (Belgium), by the Belgian Federal Science Policy 
Office through the Interuniversity Attraction Pole P6/11, by IISN-Belgium, by “Communauté française de Belgique-Actions de Recherche Concertées", and by Fondecyt Projects no. 1085322 and no. 1090753.

\section{References}

[1] H. Bondi, M. G. van der Burg, and A. W. Metzner, "Gravitational waves in general relativity. VII. Waves from axi-symmetric isolated systems," Proceedings of the Royal Society A, vol. 269, no. 1336, pp. 21-52, 1962.

[2] R. K. Sachs, "Gravitational waves in general relativity. 8. Waves in asymptotically flat space-times," Proceedings of the Royal Society A, vol. 270, pp. 103-126, 1962.

[3] E. T. Newman and T. W. J. Unti, "Behavior of asymptotically flat empty spaces," Journal of Mathematical Physics, vol. 3, no. 5, pp. 891-901, 1962.

[4] R. K. Sachs, "Asymptotic symmetries in gravitational theory," Physical Review, vol. 128, pp. 2851-2864, 1962.

[5] G. Barnich and C. Troessaert, "Symmetries of asymptotically flat four-dimensional spacetimes at null infinity revisited," Physical Review Letters, vol. 105, no. 11, Article ID 111103, 2010.

[6] G. Barnich and C. Troessaert, "Aspects of the BMS/CFT correspondence," Journal of High Energy Physics, vol. 1005, article 062, 2010.

[7] G. Barnich and C. Troessaert, "Supertranslations call for superrotations," Proceedings of Science CNCFG, 010, 2010, http:/ /arxiv.org/abs/1102.4632.

[8] R. Geroch, "Asymptotic structure of space-time," in Symposium on the Asymptotic Structure of SpaceTime, P. Esposito and L. Witten, Eds., pp. 1-105, Plenum, New York, NY, USA, 1977.

[9] R. Penrose, "Asymptotic properties of fields and space-times," Physical Review Letters, vol. 10, no. 2, pp. 66-68, 1963.

[10] C. Imbimbo, A. Schwimmer, S. Theisen, and S. Yankielowicz, "Diffeomorphisms and holographic anomalies," Classical and Quantum Gravity, vol. 17, no. 5, pp. 1129-1138, 2000.

[11] G. Barnich, "A note on gauge systems from the point of view of Lie algebroids," AIP Conference Proceedings, vol. 1307, pp. 7-18, 2010.

[12] J. Foster, "Conformal structure of $\mathrm{i}^{+}$and asymptotic symmetry. I. Definitions and local theory," Journal of Physics A, vol. 11, no. 1, pp. 93-102, 1978.

[13] J. Foster, "Asymptotic symmetry and the global structure of future null infinity," International Journal of Theoretical Physics, vol. 26, pp. 1107-1124, 1987.

[14] E. Newman and R. Penrose, "An approach to gravitational radiation by a method of spin coefficients," Journal of Mathematical Physics, vol. 3, no. 3, pp. 566-578, 1962.

[15] E. P. Newman and K. P. Tod, "Asymptotically flat space-times," in General Relativity and Gravitation. 100 Years after the Birth of Albert Einstein. Volume 2, pp. 1-36, Plenum Press, 1980.

[16] G. Barnich and C. Troessaert, "BMS charge algebra," Journal of High Energy Physics, vol. 1112, article 105, 2011.

[17] R. Penrose, "Conformal treatment of infinity," in Relativity, Groups and Topology: Les Houches 1963, B. D. C. DeWitt, Ed., pp. 563-584, Gordon and Breach, 1964.

[18] R. Penrose, "Zero rest mass fields including gravitation: asymptotic behavior," Proceedings of the Royal Society A, vol. 284, pp. 159-203, 1965.

[19] L. A. Tamburino and J. H. Winicour, "Gravitational fields in finite and conformal Bondi frames," Physical Review, vol. 150, no. 4, pp. 1039-1053, 1966.

[20] R. Penrose, "Relativistic symmetry groups," in Group Theory in Non-Linear Problems, A. O. Barut, Ed., pp. 1-58, Reidel Publishing Company, Dodrecht, The Netherlands, 1974.

[21] B. Schmidt, M. Walker, and P. Sommers, "A characterization of the Bondi-Metzner-Sachs group," General Relativity and Gravitation, vol. 6, no. 5, pp. 489-497, 1975.

[22] S. J. Fletcher and A. W. C. Lun, "The Kerr spacetime in generalized Bondi-Sachs coordinates," Classical and Quantum Gravity, vol. 20, no. 19, pp. 4153-4167, 2003.

[23] J. A. V. Kroon, "A comment on the outgoing radiation condition for the gravitational field and the peeling theorem," General Relativity and Gravitation, vol. 31, no. 8, pp. 1219-1224, 1999.

[24] E. T. Newman and R. Penrose, "Note on the Bondi-Metzner-Sachs group," Journal of Mathematical Physics, vol. 7, no. 5, pp. 863-870, 1966. 
[25] R. Penrose and W. Rindler, Spinors and Space-Time, Volume 2: Spinor and Twistor Methods in Space-Time Geometry, Cambridge University Press, 1986.

[26] A. Held, E. T. Newman, and R. Posadas, "The Lorentz group and the sphere," Journal of Mathematical Physics, vol. 11, no. 11, pp. 3145-3154, 1970.

[27] T. Dray and M. Streubel, "Angular momentum at null infinity," Classical and Quantum Gravity, vol. 1, no. 1, pp. 15-26, 1984.

[28] R. Penrose, "Quasi-local mass and angular momentum in general relativity," Proceedings of the Royal Society A, vol. 381, no. 1780, pp. 53-63, 1982.

[29] E. H. Saidi and M. Zakkari, "Harmonic distributions, Diff(S2), and Virasoro algebra," Tech. Rep. IC/90/257, ICTP, 1990. 


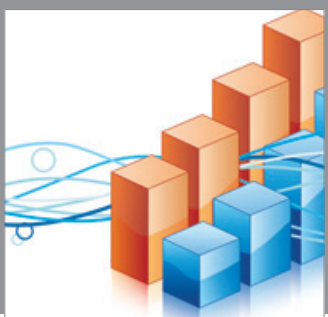

Advances in

Operations Research

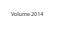

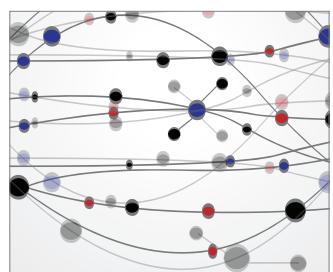

\section{The Scientific} World Journal
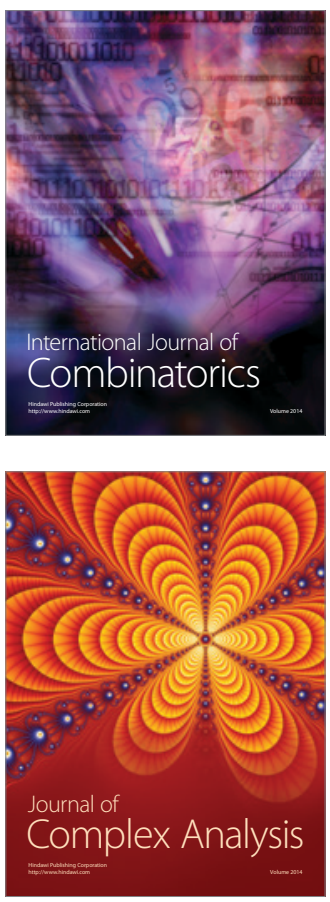

International Journal of

Mathematics and

Mathematical

Sciences
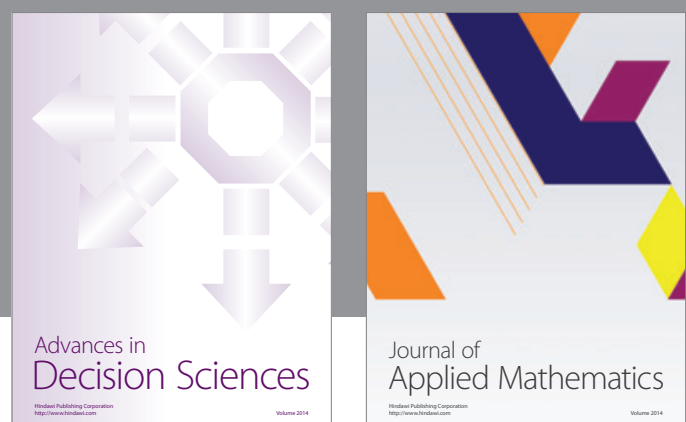

Journal of

Applied Mathematics
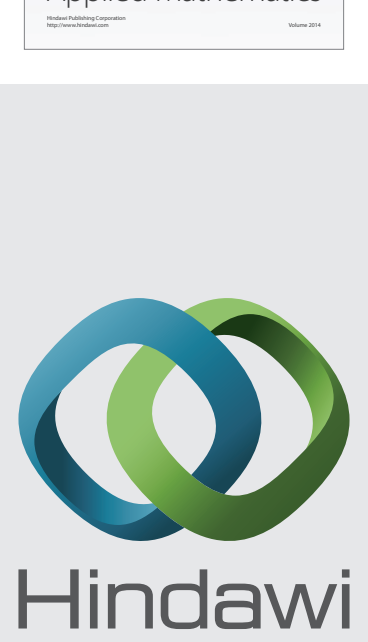

Submit your manuscripts at http://www.hindawi.com
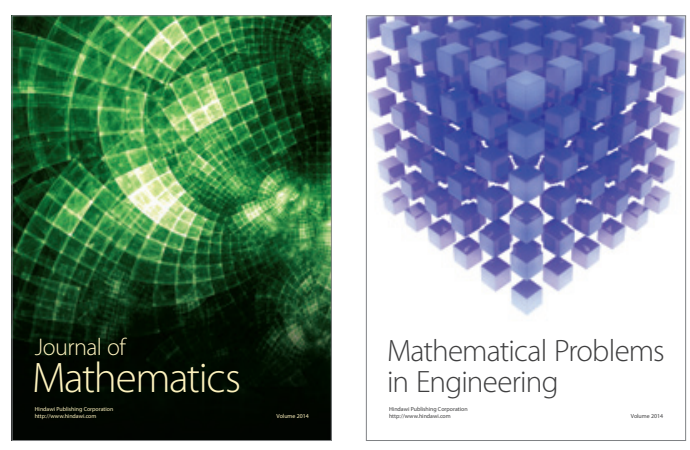

Mathematical Problems in Engineering
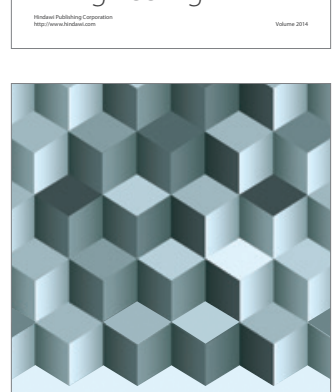

Journal of

Function Spaces
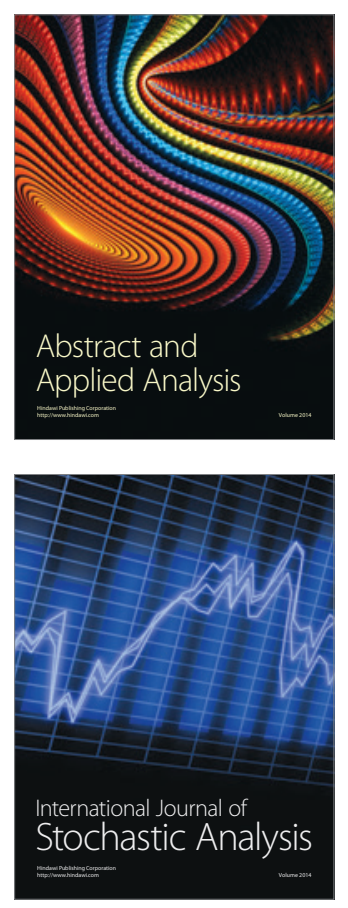

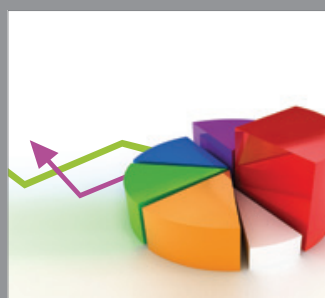

ournal of

Probability and Statistics

Promensencen
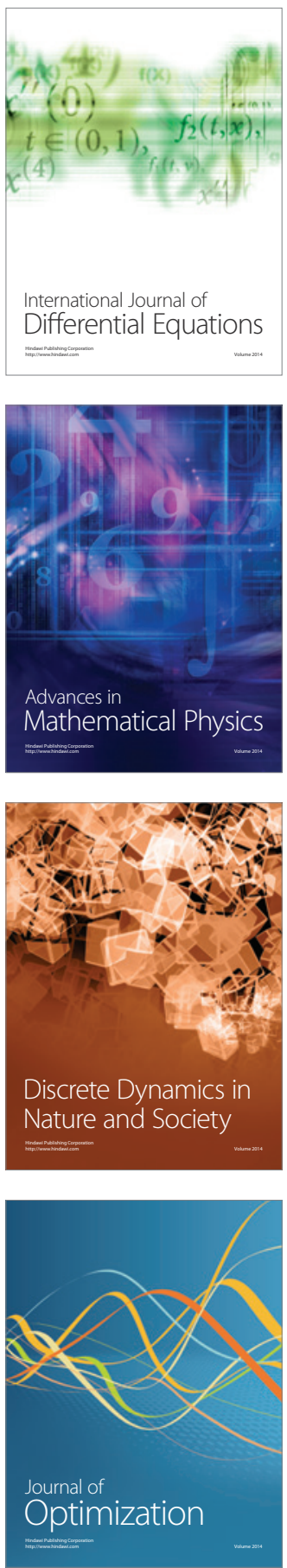